\title{
Hypoexponential Distribution with Different Parameters
}

\author{
Khaled Smaili ${ }^{1}$, Therrar Kadri ${ }^{2}$, Seifedine Kadry ${ }^{3}$ \\ ${ }^{1}$ Department of Applied Mathematics, Faculty of Sciences, Lebanese University, Zahle, Lebanon \\ ${ }^{2}$ Department of Mathematics, Faculty of Sciences, Beirut Arab University, Beirut, Lebanon \\ ${ }^{3}$ School of Engineering, American University of the Middle East, Eguaila, Kuwait \\ Email: ksmeily@hotmail.com, therrar@hotmail.com, skadry@gmail.com
}

Received January 27, 2013; revised March 4, 2013; accepted March 11, 2013

Copyright (C) 2013 Khaled Smaili et al. This is an open access article distributed under the Creative Commons Attribution License, which permits unrestricted use, distribution, and reproduction in any medium, provided the original work is properly cited.

\begin{abstract}
The Hypoexponential distribution is the distribution of the sum of $n \geq 2$ independent Exponential random variables. This distribution is used in moduling multiple exponential stages in series. This distribution can be used in many domains of application. In this paper we consider the case of $n$ exponential Random Variable having distinct parameters. Using convolution, some properties of Laplace transform and the moment generating function, we analyse this case and give new properties and identities. Moreover, we shall study particular cases when $\alpha_{i}$ are arithmetic and geometric.
\end{abstract}

Keywords: Hypoexponential Distribution; pdf; Convolution; Laplace Transform; Moment Generating Function; Expectation; Partial Fraction Expansion

\section{Introduction}

The Random Variable (RV) plays an important role in modeling many events $[1,2]$. In particular the sum of exponential random has important applications in the modeling in many domains such as communications and computer science [3,4], Markov process [5,6], insurance $[7,8]$ and reliability and performance evaluation $[4,5,9$, 10]. Nadarajah [11], presented a review of some results on the sum of random variables.

Many processes in nature can be divided into sequential phases. If the time the process spends in each phase is independent and exponentially distributed, then the overall time is hypoexponentially distributed. The service times for input-output operations in a computer system often possess this distribution. The probability density function (pdf) and cummulative distribution function (cdf) of the hypoexponential with distinct parameters were presented by many authors $[5,12,13]$. Moreover, in the domain of reliability and performance evaluation of systems and software many authors used the geometric and arithmetic parameters such as $[10,14,15]$.

In this paper we study the hypoexponential distribution in the case of $n$ independent exponential R. V. with distinct parameters $\alpha_{i} \neq \alpha_{j}$ for $i \neq j$, written as hypoexp $\left(\alpha_{1}, \alpha_{2}, \cdots, \alpha_{n}\right)$. We use in our work the properties of convolution, Laplace transform and moment generating function in finding the $k^{\text {th }}$ derivative of the pdf of this sum and the moment of this distribution of order $k$. In addition, we deduce some new equalities related to these parameters. Also we shall study the case when the parameters form an arithmetic and geometric sequence considered by $[10,14,15]$ and find some new results.

\section{Definitions and Notations}

Let $X_{1}, X_{2}, \cdots, X_{n}$ be independent exponential random variables with different respective parameters $\alpha_{i}$, $i=1,2, \cdots, n$, written as $X_{i} \sim \operatorname{Exp}\left(\alpha_{i}\right)$. We define the random variable

$$
S_{n}=\sum_{i=1}^{n} X_{i}
$$

to be the Hypoexponential random variable with parameters $\alpha_{i}, i=1,2, \cdots, n$, written as

$$
S_{n} \sim \operatorname{hypoexp}\left(\alpha_{1}, \alpha_{2}, \cdots, \alpha_{n}\right)
$$

Some notations used throughout the paper.

$X_{i}: \operatorname{Exp}\left(\alpha_{i}\right)$.

$S_{n}:$ hypoexp $\left(\alpha_{1}, \alpha_{2}, \cdots, \alpha_{n}\right)$.

$f_{X}$ : The pdf of the random variable $X$.

$F_{X}$ : The cdf of the random variable $X$.

$f_{X}^{(k)}:$ The $k^{\text {th }}$ derivative of the pdf $f_{X}$. 
$\mathcal{L}\{\cdot\}$ : Laplace-Stieltjes Transform.

$\mathcal{L}^{-1}\{\cdot\}$ : Laplace Inverse.

$\Phi_{X}(t)$ : The moment generating function of $X$.

$E\left[X^{k}\right]:$ The moment of order $k$ of the $\operatorname{RV} X$.

$\alpha: \prod_{i=1}^{n} \alpha_{i}$ product of all parameters.

$P_{i}: \prod_{j=1, j \neq i}^{n}\left(1-\frac{\alpha_{i}}{\alpha_{j}}\right)$.

$\gamma_{i}: \prod_{j=1, j \neq i}^{n}\left(\alpha_{j}-\alpha_{i}\right)$.

$E_{k}:\left\{\left(l_{1}, \cdots, l_{n}\right) / 0 \leq l_{i} \leq k ; \sum_{i=1}^{n} l_{i}=k ; 1 \leq i \leq n\right\}, E_{0}=0$.

\section{Applications on pdf and cdf Using Laplace Transform}

The pdf and cdf of the hypoexponential with distinct parameters were presented by many authors [2,7,11-13]. We shall state in thoerem 1 and propostion 1 these results and provide another proof using Laplace transform. Next, we give some new properties of its pdf, where new identities are obtained.

Theorem 1. Let $n \geq 2$ and $t>0$. Then

$$
f_{S_{n}}(t)=\sum_{i=1}^{n} \frac{f_{X_{i}}(t)}{P_{i}}
$$

and

$$
F_{S_{n}}(x)=1-\sum_{i=1}^{n} \frac{\mathrm{e}^{-\alpha_{i} x}}{P_{i}} I_{(0, \infty)}(x) .
$$

Proof. We have

$$
\mathcal{L}\left\{f_{X_{i}}(x)\right\}=\frac{\alpha_{i}}{s+\alpha_{i}},
$$

where $s>\max \left\{-\alpha_{i}\right\}$ for $i=1,2, \cdots, n$. Since $X_{i}$ are independent then $f_{S_{n}}(t)$ is the convolutions of $f_{X_{i}}$, $i=1,2, \cdots, n$ written as

$$
f_{S_{n}}(t)=\left(f_{X_{1}} * f_{X_{2}} * \ldots * f_{X_{n}}\right)(t)
$$

and the Laplace transform of convolution of functions is the product of their Laplace transform, thus

$$
\begin{aligned}
\mathcal{L}\left\{f_{S_{n}}(t)\right\} & =\prod_{i=1}^{n} \mathcal{L}\left\{f_{X_{i}}(t)\right\} \\
& =\prod_{i=1}^{n} \frac{\alpha_{i}}{s+\alpha_{i}}=\alpha \prod_{i=1}^{n} \frac{1}{s+\alpha_{i}}
\end{aligned}
$$

where $s>\max \left\{-\alpha_{i}\right\}$. However, by Heaviside Expansion Theorem [16], for distinct poles gives that

$$
\mathcal{L}\left\{f_{s_{n}}(t)\right\}=\alpha \sum_{i=1}^{n} \frac{A_{i}}{s+\alpha_{i}},
$$

where

$$
A_{i}=\frac{1}{\prod_{j=1, j \neq i}^{n}\left(\alpha_{j}-\alpha_{i}\right)}
$$

Therefore,

$$
\begin{aligned}
f_{S_{n}}(t) & =\mathcal{L}^{-1}\left\{\alpha \sum_{i=1}^{n} \frac{A_{i}}{s+\alpha_{i}}\right\} \\
& =\alpha \sum_{i=1}^{n} A_{i} \mathrm{e}^{-\alpha_{i} t} I_{(0, \infty)}(t) .
\end{aligned}
$$

But $\alpha A_{i}=\frac{\alpha_{i}}{P_{i}}$. Thus

$$
f_{S_{n}}(t)=\sum_{i=1}^{n} \frac{f_{X_{i}}(t)}{P_{i}} .
$$

On the other hand we have

$$
\begin{aligned}
F_{S_{n}}(x) & =\int_{0}^{x} \sum_{i=1}^{n} \frac{f_{X_{i}}(t) \mathrm{d} t}{P_{i}} \\
& =\sum_{i=1}^{n} \frac{1}{P_{i}} F_{X_{i}}(x)=\sum_{i=1}^{n} \frac{1-\mathrm{e}^{-\alpha_{i} x}}{P_{i}} .
\end{aligned}
$$

But $\lim _{x \rightarrow \infty} F_{S_{n}}(x)=1$, then $\sum_{i=1}^{n} \frac{1}{P_{i}}=1$ and we conclude that

$$
F_{S_{n}}(x)=1-\sum_{i=1}^{n} \frac{\mathrm{e}^{-\alpha_{i} x}}{P_{i}} I_{(0, \infty)}(x) .
$$

Next we shall discuss the $k^{\text {th }}$ derivative of $f_{S_{n}}(t)$ and many equalities are obtained concerning $P_{i}$ form and some similar forms.

We start by noting from the previous proof that $\sum_{i=1}^{n} \frac{1}{P_{i}}=1$. Here, we shall state another simple proof using Laplace transform.

Proposition 1. Let $n \geq 2$. Then

$$
\sum_{i=1}^{n} \frac{1}{P_{i}}=1 \text {. }
$$

Proof. We have from Equation (1),

$$
\mathcal{L}\left\{f_{s_{n}}(t)\right\}=\prod_{i=1}^{n}\left(\frac{\alpha_{i}}{s+\alpha_{i}}\right)
$$

where $s>\max \left\{-\alpha_{i}\right\}, i=1,2, \cdots, n$. But from Theorem 1 ,

$$
f_{S_{n}}(t)=\sum_{i=1}^{n} \frac{f_{X_{i}}(t)}{P_{i}}
$$

and

$$
\begin{aligned}
\mathcal{L}\left\{f_{S_{n}}(t)\right\} & =\sum_{i=1}^{n} \frac{\mathcal{L}\left\{f_{X_{i}}(t)\right\}}{P_{i}} \\
& =\sum_{i=1}^{n} \frac{\alpha_{i}}{P_{i}\left(s+\alpha_{i}\right)} I_{(0, \infty)}(t) .
\end{aligned}
$$

Hence, $\prod_{i=1}^{n}\left(\frac{\alpha_{i}}{s+\alpha_{i}}\right)=\sum_{i=1}^{n} \frac{\alpha_{i}}{P_{i}\left(s+\alpha_{i}\right)}$. For $s=0$, 
$\prod_{i=1}^{n}\left(\frac{\alpha_{i}}{\alpha_{i}}\right)=\sum_{i=1}^{n} \frac{\alpha_{i}}{P_{i}\left(\alpha_{i}\right)}$. Therefore, $\sum_{i=1}^{n} \frac{1}{P_{i}}=1$.

Lemma 1. Let $n \geq 2$. Then

$$
\mathcal{L}\left\{f_{s_{n}}^{(k)}(t)\right\}=s^{k} \prod_{i=1}^{n}\left(\frac{\alpha_{i}}{s+\alpha_{i}}\right)
$$

for $0 \leq k \leq n-1$.

Proof. The proof is done by induction. For $k=0$, we have from Equation (1)

$$
\mathcal{L}\left\{f_{S_{n}}(t)\right\}=\prod_{i=1}^{n}\left(\frac{\alpha_{i}}{s+\alpha_{i}}\right) .
$$

However, by Initial Value Theorem, we have

$$
\begin{aligned}
\lim _{t \rightarrow 0} f_{S_{n}}(t) & =\lim _{s \rightarrow+\infty} s \mathcal{L}\left\{f_{S_{n}}(t)\right\} \\
& =\lim _{s \rightarrow+\infty} s \prod_{i=1}^{n}\left(\frac{\alpha_{i}}{s+\alpha_{i}}\right)=0
\end{aligned}
$$

and for $k=1$ we have

$$
\begin{aligned}
\mathcal{L}\left\{f_{s_{n}}^{(1)}(t)\right\} & =s \mathcal{L}\left\{f_{s_{n}}(t)\right\}-f_{s_{n}}\left(0^{+}\right) \\
& =s \mathcal{L}\left\{f_{s_{n}}(t)\right\}=s \prod_{i=1}^{n}\left(\frac{\alpha_{i}}{s+\alpha_{k}}\right) .
\end{aligned}
$$

Moreover

$$
\mathcal{L}\left\{f_{S_{n}}^{(k+1)}(t)\right\}=s \mathcal{L}\left\{f_{S_{n}}^{(k)}(t)\right\}-f_{S_{n}}^{(k)}\left(0^{+}\right)
$$

Continuing in the same manner till the $(n-1)^{\text {th }}$ derivative, we obtain the result.

In the following propostion we shall prove that the first $(n-2)^{\text {th }}$ derivative of the pdf of $S_{n}$ are zeros, which verifies the fact that the coefficient of variation of the hypoexponential distribution is less than one unlike the hyperexponential distribution that have the coefficient of variation greater than 1 .

Proposition 2. Let $n \geq 2$. Then

$$
\lim _{t \rightarrow 0} f_{S_{n}}^{(k)}(t)=\left\{\begin{array}{l}
0, \text { if } 0 \leq k \leq n-2 \\
\alpha, \text { if } k=n-1
\end{array}\right.
$$

Proof. Let $n \geq 2$, we have from Lemma 1,

$$
\mathcal{L}\left\{f_{s_{n}}^{(k)}(t)\right\}=s^{k} \prod_{i=1}^{n}\left(\frac{\alpha_{i}}{s+\alpha_{i}}\right)
$$

for $0 \leq k \leq n-1$ and from Initial Value Theorem, we have

$$
\begin{aligned}
\lim _{t \rightarrow 0} f_{S_{n}}^{(k)}(t) & =\lim _{s \rightarrow+\infty} s \mathcal{L}\left\{f_{S_{n}}^{(k)}(t)\right\}=\lim _{s \rightarrow+\infty} \frac{s^{k+1}}{s^{n}} \alpha \\
& =\lim _{s \rightarrow+\infty} \frac{1}{s^{n-k-1}} \alpha=\left\{\begin{array}{l}
0, \text { if } n-k-1 \geq 1 \\
\alpha, \text { if } n-k-1=0
\end{array}\right.
\end{aligned}
$$

Corollary 1. Let $n \geq 2$. Then

$$
\sum_{i=1}^{n} \frac{\alpha_{i}^{k}}{P_{i}}= \begin{cases}0, & \text { if } 1 \leq k \leq n-1 \\ (-1)^{n-1} \alpha, & \text { if } k=n\end{cases}
$$

Proof. We have $f_{X_{i}}(t)=\alpha_{i} \mathrm{e}^{-\alpha_{i} t} I_{(0, \infty)}(t)$. Then the $r^{\text {th }}$ derivative of $f_{X_{i}}$ is

$$
f_{X_{i}}^{(r)}(t)=(-1)^{r} \alpha_{i}^{r+1} \mathrm{e}^{-\alpha_{i} t} I_{(0, \infty)}(t) .
$$

However, from Theorem 1,

$$
f_{S_{n}}(t)=\sum_{i=1}^{n} \frac{f_{X_{i}}(t)}{P_{i}},
$$

then

$$
f_{S_{n}}^{(r)}(t)=\sum_{i=1}^{n} \frac{f_{X_{i}}^{(r)}(t)}{P_{i}}=\sum_{i=1}^{n}(-1)^{r} \frac{\alpha_{i}^{r+1} \mathrm{e}^{-\alpha_{i} t}}{P_{i}} I_{(0, \infty)}
$$

and

$$
\lim _{t \rightarrow 0} f_{S_{n}}^{(r)}(t)=(-1)^{r} \sum_{i=1}^{n} \frac{\alpha_{i}^{r+1}}{P_{i}} .
$$

By Proposition 2, we obtain that

$$
\sum_{i=1}^{n} \frac{\alpha_{i}^{r+1}}{P_{i}}= \begin{cases}0, & \text { if } 0 \leq r \leq n-2 \\ (-1)^{r} \alpha, & \text { if } r=n-1\end{cases}
$$

By replacing $r+1$ with $k$ we obtain the result.

\section{Applications on pdf and cdf Using Moment Generating Function}

In the previous section we saw the use of Laplace properties in the proofs of the theorems and propositions. In a similar manner, in this section we use the moment genrating function to obtain more new related results. A new form of the moment generating function of $S_{n}$ and the moment of $S_{n}$ of order $k$ is given. Moreover, we deduce more new related equalities concerning $P_{i}$ and higher order derivatives of pdf of $S_{n}$.

Proposition 3. Let $n \geq 2$. Then

$$
\Phi_{S_{n}}(t)=\sum_{i=1}^{n} \frac{\Phi_{X_{i}}(t)}{P_{i}} .
$$

Proof. We have

$$
\Phi_{S_{n}}(t)=E\left[\mathrm{e}^{t S_{n}}\right]=\int_{-\infty}^{+\infty} \mathrm{e}^{t x} f_{S_{n}}(x) \mathrm{d} x
$$

and from Theorem 1,

$$
f_{S_{n}}(t)=\sum_{i=1}^{n} \frac{f_{X_{i}}(t)}{P_{i}},
$$

then

$$
\Phi_{S_{n}}(t)=\sum_{i=1}^{n} \frac{1}{P_{i}} \int_{-\infty}^{+\infty} \mathrm{e}^{t x} f_{X_{i}}(x) \mathrm{d} x=\sum_{i=1}^{n} \frac{\Phi_{X_{i}}(t)}{P_{i}} .
$$


Proposition 4. Let $n \geq 2$ and $k \geq 0$. Then

$$
E\left[S_{n}^{k}\right]=\sum_{i=1}^{n} \frac{k !}{P_{i} \alpha_{i}^{k}}
$$

Proof. We have from Proposition 3,

$$
\Phi_{S_{n}}(t)=\sum_{i=1}^{n} \frac{\Phi_{X_{i}}(t)}{P_{i}} .
$$

Then

$$
\frac{\mathrm{d}^{k} \Phi_{S_{n}}(t)}{\mathrm{d} t^{k}}=\sum_{i=1}^{n} \frac{1}{P_{i}} \frac{\mathrm{d}^{k} \Phi_{X_{i}}(t)}{\mathrm{d} t^{k}}
$$

and

$$
\left.\frac{\mathrm{d}^{k} \Phi_{S_{n}}(t)}{\mathrm{d} t^{k}}\right|_{t=0}=\left.\sum_{i=1}^{n} \frac{1}{P_{i}} \frac{\mathrm{d}^{k} \Phi_{X_{i}}(t)}{\mathrm{d} t^{k}}\right|_{t=0}
$$

which gives $E\left[S_{n}^{k}\right]=\sum_{i=1}^{n} \frac{E\left[X_{i}^{k}\right]}{P_{i}}$. But $E\left[X_{i}^{k}\right]=\frac{k !}{\alpha_{i}^{k}}$.

Thus we obtain the result.

Next, we shall use the Proposition 3 and 4 to find other identities on $P_{i}$ and higher orders for $f_{s_{n}}^{(k)}(t)$. We start by noting that $\Phi_{S_{n}}(0)=1$ and by taking $t=0$ in Proposition 3, we again obtain the result in Proposition 1, that is $\sum_{i=1}^{n} \frac{1}{P_{i}}=1$.

Proposition 5. Let $n \geq 2$ and $k \geq 0$. Then

where

$$
\sum_{i=1}^{n} \frac{1}{P_{i} \alpha_{i}^{k}}=\sum_{E_{k}} \frac{1}{\alpha_{1}^{l_{1}} \alpha_{2}^{l_{2}} \cdots \alpha_{n}^{l_{n}}} .
$$

$$
E_{k}=\left\{\left(l_{1}, \cdots, l_{n}\right) / 0 \leq l_{i} \leq k ; \sum_{i=1}^{n} l_{i}=k ; 1 \leq i \leq n\right\} .
$$

Note that we may write

$$
\sum_{E_{k}} \frac{1}{\alpha_{1}^{l_{1}} \alpha_{2}^{l_{2}} \cdots \alpha_{n}^{l_{n}}}=\sum_{I_{k}} \frac{1}{\alpha_{i_{1}} \alpha_{i_{2}} \alpha_{i_{3}} \cdots \alpha_{i_{k}}},
$$

where

$$
I_{k}=\left\{\left(i_{1}, \cdots, i_{k}\right) / 1 \leq i_{1} \leq i_{2} \leq \cdots \leq i_{k} \leq n\right\} .
$$

However $E_{k}$ and $I_{k}$ are equivalent representing a set of combination with repetition having $\left(\begin{array}{c}n+k-1 \\ k\end{array}\right)$ possibilities and $E_{0}=I_{0}=0$, thus the above summation (3) shall be 1.

Proof. Let $k \geq 0$ and $n \geq 2$. We have

$$
E\left[S_{n}^{k}\right]=E\left[\left(X_{1}+X_{2}+\cdots+X_{n}\right)^{k}\right]
$$

and using multinomial expansion formula, we obtain

$$
E\left[S_{n}^{k}\right]=E\left[\sum_{E_{k}} \frac{k !}{l_{1} ! l_{2} ! \cdots l_{n} !}\left(X_{1}^{l_{1}} X_{2}^{l_{2}} \cdots X_{n}^{l_{n}}\right)\right] .
$$

Knowing that expectation is linear and $X_{i}$, $i=1,2, \cdots, n$ are independent with

$$
E\left[X_{i}^{l_{i}}\right]=\frac{l_{i} !}{\alpha_{i}^{l_{i}}},
$$

then

$$
E\left[S_{n}^{k}\right]=\sum_{E_{k}} \frac{k !}{\alpha_{1}^{l_{1}} \alpha_{2}^{l_{2}} \cdots \alpha_{n}^{l_{n}}} .
$$

Since from Proposition 4,

$$
E\left[S_{n}^{k}\right]=\sum_{i=1}^{n} \frac{k !}{P_{i} \alpha_{i}^{k}} .
$$

Therefore,

$$
\sum_{i=1}^{n} \frac{1}{P_{i} \alpha_{i}^{k}}=\sum_{E_{k}} \frac{1}{\alpha_{1}^{l_{1}} \alpha_{2}^{l_{2}} \cdots \alpha_{n}^{l_{n}}} .
$$

The following corollary is direct consequence of Proposition 5 and Equation (4), taking $k=0,1$ and 2 respectively.

Corollary 2. Let $n \geq 2$. Then

1) $\sum_{i=1}^{n} \frac{1}{P_{i}}=1$.

2) $\sum_{i=1}^{n} \frac{1}{P_{i} \alpha_{i}}=\sum_{i=1}^{n} \frac{1}{\alpha_{i}}$ and $E\left[S_{n}\right]=\sum_{i=1}^{n} \frac{1}{\alpha_{i}}$.

3) $\sum_{i=1}^{n} \frac{1}{P_{i} \alpha_{i}^{2}}=\sum_{1 \leq i \leq j \leq n} \frac{1}{\alpha_{i} \alpha_{j}}$ and $E\left[S_{n}^{2}\right]=\sum_{1 \leq i \leq j \leq n} \frac{2 !}{\alpha_{i} \alpha_{j}}$.

In Proposition 2, we found the first $(n-1)^{\text {th }}$ derivative of $f_{S_{n}}$ at 0 , However to find higher order derivaties we recall Equation (2), that shows a direct relation between the $k^{\text {th }}$ derivative $f_{S_{n}}$ and $\sum_{i=1}^{n} \frac{\alpha_{i}^{k}}{P_{i}}$. Hence, in the next propostion we shall use Propostion 5, to find an equation for $\sum_{i=1}^{n} \frac{\alpha_{i}^{k}}{P_{i}}$ by finding a relation between $\operatorname{hypoexp}\left(\alpha_{1}, \alpha_{2}, \cdots, \alpha_{n}\right)$ and hypoexp $\left(\frac{1}{\alpha_{1}}, \frac{1}{\alpha_{2}}, \cdots, \frac{1}{\alpha_{n}}\right)$.

Proposition 6. Let $n \geq 2$ and $k \geq n$. Then

$$
\sum_{i=1}^{n} \frac{\alpha_{i}^{k}}{P_{i}}=(-1)^{n-1} \alpha \sum_{E_{k-n}} \alpha_{1}^{l_{1}} \alpha_{2}^{l_{2}} \cdots \alpha_{n}^{l_{n}} .
$$

Proof. Let $n \geq 2, \beta_{i}=\frac{1}{\alpha_{i}}, i=1,2, \cdots, n$ and

$C_{n} \sim \operatorname{hypoexp}\left(\beta_{1}, \beta_{2}, \cdots, \beta_{n}\right)$. Then by Theorem 1 , the pdf of $C_{n}$ is

$$
f_{C_{n}}(t)=\sum_{i=1}^{n} \frac{f_{Y_{i}}(t)}{B_{i}}
$$


where $Y_{i} \sim \operatorname{Exp}\left(\beta_{i}\right)$ and $B_{i}=\prod_{j=1, j \neq i}^{n}\left(1-\frac{\beta_{i}}{\beta_{j}}\right)$.

Next, we shall find $P_{i}$ in terms of $B_{i}$. We have

$$
\begin{aligned}
P_{i} & =\prod_{j=1, j \neq i}^{n}\left(1-\frac{\alpha_{i}}{\alpha_{j}}\right)=\prod_{j=1, j \neq i}^{n}\left(1-\frac{\beta_{j}}{\beta_{i}}\right) \\
& =\frac{(-1)^{n-1} \prod_{j=1, j \neq i}^{n}\left(\beta_{j}-\beta_{i}\right)}{\beta_{i}^{n-1}},
\end{aligned}
$$

multiplying in the numerator and denominator by $\prod_{j=1, j \neq i}^{n}\left(\beta_{j}\right)$, we obtain $P_{i}=(-1)^{n-1} \frac{\beta}{\beta_{i}^{n}} B_{i}$ where $\beta=\prod_{j=1}^{n}\left(\beta_{j}\right)$. Hence, we may write

$$
\sum_{i=1}^{n} \frac{\alpha_{i}^{k}}{P_{i}}=\frac{(-1)^{n-1}}{\beta} \sum_{i=1}^{n} \frac{1}{\beta_{i}^{k-n} B_{i}} .
$$

But, for $k \geq n$ Proposition 5 gives that

$$
\sum_{i=1}^{n} \frac{1}{\beta_{i}^{k-n} B_{i}}=\sum_{E_{k-n}} \frac{1}{\beta_{1}^{l_{1}} \beta_{2}^{l_{2}} \cdots \beta_{n}^{I_{n}}} .
$$

Therefore,

$$
\begin{aligned}
\sum_{i=1}^{n} \frac{\alpha_{i}^{k}}{P_{i}} & =\frac{(-1)^{n-1}}{\beta} \sum_{E_{k-n}} \frac{1}{\beta_{1}^{l_{1}} \beta_{2}^{l_{2}} \cdots \beta_{n}^{l_{n}}} \\
& =(-1)^{n-1} \alpha \sum_{E_{k-n}} \alpha_{1}^{l_{1}} \alpha_{2}^{l_{2}} \cdots \alpha_{n}^{l_{n}} .
\end{aligned}
$$

Proposition 7. Let $n \geq 2$ and $k \geq n-1$. Then

$$
\lim _{t \rightarrow 0} f_{S_{n}}^{(k)}(t)=(-1)^{k+n-1} \alpha \sum_{E_{k-n+1}} \alpha_{1}^{l_{1}} \alpha_{2}^{l_{2}} \cdots \alpha_{n}^{l_{n}} .
$$

Proof. We have from Equation (2),

$$
\lim _{t \rightarrow 0} f_{S_{n}}^{(k)}(t)=(-1)^{k} \sum_{i=1}^{n} \frac{\alpha_{i}^{k+1}}{P_{i}} .
$$

and from Proposition 6,

$$
\sum_{i=1}^{n} \frac{\alpha_{i}^{k+1}}{P_{i}}=(-1)^{n-1} \alpha \sum_{E_{k-n+1}} \alpha_{1}^{l_{1}} \alpha_{2}^{l_{2}} \cdots \alpha_{n}^{l_{n}} .
$$

for $k \geq n-1$. Then,

$$
\lim _{t \rightarrow 0} f_{S_{n}}^{(k)}(t)=(-1)^{k+n-1} \alpha \sum_{E_{k-n+1}} \alpha_{1}^{l_{1}} \alpha_{2}^{l_{2}} \cdots \alpha_{n}^{l_{n}} .
$$

Many authors used the identity

$$
\sum_{i=1}^{n}\left[\frac{1}{\prod_{j=1, j \neq i}^{n}\left(\alpha_{j}-\alpha_{i}\right)}\right]=0
$$

and proved it in many long and complicated methods. Here we shall submit a more simple prove. In addition, we shall find more related identities using the above results.

Proposition 8. Let $n \geq 2$. Then

$$
\sum_{i=1}^{n}\left[\frac{1}{\prod_{j=1, j \neq i}^{n}\left(\alpha_{j}-\alpha_{i}\right)}\right]=0 .
$$

Proof. Let $n \geq 2$. By Corollary 1, taking $i=1$ we have $i \leq n-1$, then

$$
\sum_{i=1}^{n} \frac{\alpha_{i}}{P_{i}}=0 .
$$

However,

$$
\begin{aligned}
\sum_{i=1}^{n} \frac{\alpha_{i}}{P_{i}} & =\sum_{i=1}^{n} \frac{\alpha_{i} \prod_{j=1, j \neq i}^{n}\left(\alpha_{j}\right)}{\prod_{j=1, j \neq i}^{n}\left(\alpha_{j}-\alpha_{i}\right)} \\
& =\alpha \sum_{i=1}^{n} \frac{1}{\prod_{j=1, j \neq i}^{n}\left(\alpha_{j}-\alpha_{i}\right)}=0
\end{aligned}
$$

Therefore,

$$
\sum_{i=1}^{n}\left[\frac{1}{\prod_{j=1, j \neq i}^{n}\left(\alpha_{j}-\alpha_{i}\right)}\right]=0 .
$$

Next we shall find a more general equality using our previous results.

Proposition 9. Let $n \geq 2$. Then

$$
\begin{aligned}
& \sum_{i=1}^{n}\left[\frac{\alpha_{i}^{k}}{\prod_{j=1, j \neq i}^{n}\left(\alpha_{j}-\alpha_{i}\right)}\right] \\
& = \begin{cases}0, & \text { if } 0 \leq k \leq n-2 \\
(-1)^{n-1} \sum_{E_{k-n+1}} \alpha_{1}^{l_{1}} \alpha_{2}^{l_{2}} \cdots \alpha_{n}^{l_{n}}, & \text { if } k \geq n-1\end{cases}
\end{aligned}
$$

Proof. Let $n \geq 2$. Then,

$$
\begin{aligned}
\sum_{i=1}^{n} \frac{\alpha_{i}^{k}}{P_{i}} & =\sum_{i=1}^{n} \frac{\alpha_{i}^{k} \prod_{j=1, j \neq i}^{n}\left(\alpha_{j}\right)}{\prod_{j=1, j \neq i}^{n}\left(\alpha_{j}-\alpha_{i}\right)} \\
& =\alpha \sum_{i=1}^{n} \frac{\alpha_{i}^{k-1}}{\prod_{j=1, j \neq i}^{n}\left(\alpha_{j}-\alpha_{i}\right)}
\end{aligned}
$$

Suppose that $1 \leq k \leq n$. We have from Corollary 1 ,

$$
\sum_{i=1}^{n} \frac{\alpha_{i}^{k}}{P_{i}}= \begin{cases}0, & \text { if } 1 \leq k \leq n-1 \\ (-1)^{n-1} \alpha, & \text { if } k=n\end{cases}
$$

and Equation (5) gives that

$$
\sum_{i=1}^{n}\left[\frac{\alpha_{i}^{k-1}}{\prod_{j=1, j \neq i}^{n}\left(\alpha_{j}-\alpha_{i}\right)}\right]= \begin{cases}0, & \text { if } 1 \leq k \leq n-1 \\ (-1)^{n-1}, & \text { if } k=n\end{cases}
$$

Replace $k-1$ with $k$, we obtain the first case and 
the case when $k=n-1$, where $\sum_{E_{0}} \alpha_{1}^{l_{1}} \alpha_{2}^{l_{2}} \cdots \alpha_{n}^{l_{n}}=1$.

Now, suppose $k \geq n$. By Proposition 6,

$$
\sum_{i=1}^{n} \frac{\alpha_{i}^{k}}{P_{i}}=(-1)^{n-1} \alpha \sum_{E_{k-n}} \alpha_{1}^{l_{1}} \alpha_{2}^{l_{2}} \cdots \alpha_{n}^{l_{n}}
$$

and the Equation (5) gives that

$$
\sum_{i=1}^{n}\left[\frac{\alpha_{i}^{k-1}}{\prod_{j=1, j \neq i}^{n}\left(\alpha_{j}-\alpha_{i}\right)}\right]=(-1)^{n-1} \sum_{E_{k-n}} \alpha_{1}^{l_{1}} \alpha_{2}^{l_{2}} \cdots \alpha_{n}^{l_{n}} .
$$

Also, replace $k-1$ by $k$ we obtain the last case when $n \geq k-1$.

\section{The Main Results}

We summarize Proposition 2 and 7 in the following theorem.

Theorem 2. Let $n \geq 2$. Then

$$
\begin{aligned}
& \lim _{t \rightarrow 0} f_{S_{n}}^{(k)}(t) \\
& = \begin{cases}0, & \text { if } 0 \leq k \leq n-2 \\
(-1)^{k+n-1} \alpha \sum_{E_{k-n+1}} \alpha_{1}^{l_{1}} \alpha_{2}^{l_{2}} \cdots \alpha_{n}^{l_{n}}, & \text { if } k \geq n-1\end{cases}
\end{aligned}
$$

Also Corollary 1 and Proposition 5 and 6 can be summarized in the following theorem.

Theorem 3. Let $n \geq 2$ and $k \geq 0$. Then

1) $\sum_{i=1}^{n} \frac{\alpha_{i}^{k}}{P_{i}}= \begin{cases}0, & \text { if } 0 \leq k \leq n-1 \\ (-1)^{n-1} \alpha \sum_{E_{k-n}} \alpha_{1}^{l_{1}} \alpha_{2}^{l_{2}} \cdots \alpha_{n}^{l_{n}}, & \text { if } k \geq n\end{cases}$ and

2) $\sum_{i=1}^{n} \frac{1}{P_{i} \alpha_{i}^{k}}=\sum_{E_{k}} \frac{1}{\alpha_{1}^{l_{1}} \alpha_{2}^{l_{2}} \cdots \alpha_{n}^{l_{n}}}$.

We recall Propostion 9 in the following corollary of Theorem 3.

Corollary 3. Let $n \geq 2$. Then

$$
\begin{aligned}
& \sum_{i=1}^{n}\left[\frac{\alpha_{i}^{k}}{\prod_{j=1, j \neq i}^{n}\left(\alpha_{j}-\alpha_{i}\right)}\right] \\
& = \begin{cases}0, & \text { if } 0 \leq k \leq n-2 \\
(-1)^{n-1} \sum_{E_{k-n+1}} \alpha_{1}^{l_{1}} \alpha_{2}^{l_{2}} \cdots \alpha_{n}^{l_{n}}, & \text { if } k \geq n-1\end{cases}
\end{aligned}
$$

\section{Case of Arithmetic and Geometric Parameters}

The study of reliability and performance evaluation of systems and softwares use in general sum of independent exponential R.V. with distinct parameters. The model of Jelinski and Moranda [14], considered that the parameters changes in an arithmetic sequence $\alpha_{i}=\alpha_{i-1}+d$. Moreover, Moranda [15], considered the model when $\alpha_{i}$ changes in an geometric sequence $\alpha_{i}=\alpha_{i-1} r$. In this section, we study the hypoexponential in these two cases when the parameters are arithmetic and geometric, and we present their pdf.

\subsection{Case of Arithmetic Parameters}

We first consider the case when $\alpha_{i}, i=1,2, \cdots, n$ form an arithmetic sequence of common difference $d$.

Lemma 2. For all $1 \leq i \leq n$.

$$
\gamma_{i}=(-1)^{i-1} \frac{(n-1) !}{\left(\begin{array}{c}
n-1 \\
i-1
\end{array}\right)} d^{n-1}
$$

Proof. Suppose that $\alpha_{i}$ form an arithmetic sequence of common difference $d$. Then $\alpha_{j^{-}} \alpha_{i}=(j-i) d$. We have

$$
\gamma_{i}=\prod_{j=1, j \neq i}^{n}\left(\alpha_{j}-\alpha_{i}\right)
$$

Hence,

$$
\begin{aligned}
\gamma_{i} & =\left(\alpha_{1}-\alpha_{i}\right)\left(\alpha_{2}-\alpha_{i}\right) \cdots\left(\alpha_{i-1}-\alpha_{i}\right)\left(\alpha_{i+1}-\alpha_{i}\right) \cdots\left(\alpha_{n}-\alpha_{i}\right) \\
& =(-(i-1) d) \cdots(-2 d)(d)(2 d) \cdots((n-i) d) \\
& =(-1)^{i-1}(i-1) !(n-i) ! d^{n-1}
\end{aligned}
$$

However,

$$
(i-1) !(n-i) !=\frac{n !}{i\left(\begin{array}{c}
n \\
i
\end{array}\right)}=\frac{(n-1) !}{\left(\begin{array}{c}
n-1 \\
i-1
\end{array}\right)} .
$$

Then

$$
\gamma_{i}=(-1)^{i-1} \frac{(n-1) !}{\left(\begin{array}{c}
n-1 \\
i-1
\end{array}\right)} d^{n-1}
$$

Lemma 3. For all $1 \leq i \leq n$.

$$
\gamma_{i}=(-1)^{n-1} \gamma_{n-(i-1)} .
$$

Proof. We have from Lemma 2,

$$
\gamma_{i}=(-1)^{i-1} \frac{(n-1) !}{\left(\begin{array}{c}
n-1 \\
i-1
\end{array}\right)} d^{n-1}
$$

for all $1 \leq i \leq n$. Replace $i$ by $n-(i-1)$, we obtain

$$
\begin{aligned}
\gamma_{n-(i-1)} & =(-1)^{n-i} \frac{(n-1) !}{\left(\begin{array}{c}
n-1 \\
n-(i-1)
\end{array}\right)} d^{n-1} \\
& =(-1)^{n-i} \frac{(n-1) !}{\left(\begin{array}{c}
n-1 \\
i-1
\end{array}\right)} d^{n-1}=(-1)^{n-1} \gamma_{i} .
\end{aligned}
$$


Thus we obtain the result.

Proposition 10. Let $n \geq 2$. Then

$$
f_{S_{n}}(t)=\alpha \sum_{i=1}^{n} \frac{\mathrm{e}^{-\alpha_{i} t}}{\gamma_{i}} I_{(0, \infty)}(t),
$$

where

$$
\gamma_{i}=(-1)^{i-1} \frac{(n-1) !}{\left(\begin{array}{c}
n-1 \\
i-1
\end{array}\right)} d^{n-1}=(-1)^{n-1} \gamma_{n-(i-1)}
$$

for all $1 \leq i \leq n$.

Proof. We have from Theorem 1

$$
\begin{aligned}
f_{S_{n}}(t) & =\sum_{i=1}^{n} \frac{\alpha_{i} \mathrm{e}^{-\alpha_{i} t}}{\prod_{j=1, j \neq i}^{n}\left(1-\frac{\alpha_{i}}{\alpha_{j}}\right)} I_{(0, \infty)}(t) \\
& =\sum_{i=1}^{n} \frac{\alpha_{i} \mathrm{e}^{-\alpha_{i} t} \prod_{j=1, j \neq i}^{n}\left(\alpha_{j}\right)}{\prod_{j=1, j \neq i}^{n}\left(\alpha_{j}-\alpha_{i}\right)} I_{(0, \infty)}(t),
\end{aligned}
$$

that can be written as

$$
f_{S_{n}}(t)=\alpha \sum_{i=1}^{n} \frac{\mathrm{e}^{-\alpha_{i} t}}{\gamma_{i}} I_{(0, \infty)}(t),
$$

where $\gamma_{i}=\prod_{j=1, j \neq i}^{n}\left(\alpha_{j}-\alpha_{i}\right)$ and by the Lemmas 2 and 3 we obtain the result.

\subsection{Case of Arithmetic Parameters}

Next, we consider the case when $\alpha_{i}, i=1,2, \cdots, n$ form a geometric sequence of common ratio $r$.

Proposition 11. Let $n \geq 2$. Then

$$
f_{S_{n}}(t)=\sum_{i=1}^{n} \frac{\alpha_{i} \mathrm{e}^{-\alpha_{i} t}}{\prod_{j=1, j \neq i}^{n}\left(1-r^{i-j}\right)} I_{(0, \infty)}(t) .
$$

Proof. We have from Theorem 1,

$$
f_{S_{n}}(t)=\sum_{i=1}^{n} \frac{\alpha_{i} \mathrm{e}^{-\alpha_{i} t}}{\prod_{j=1, j \neq i}^{n}\left(1-\frac{\alpha_{i}}{\alpha_{j}}\right)} I_{(0, \infty)}(t) .
$$

Suppose now the parameter $\alpha_{i}$ form geometric sequence of common ratio $r$. Then $\alpha_{i}=\alpha_{j} r^{i-j}$ and

$$
P_{i}=\prod_{j=1, j \neq i}^{n}\left(1-\frac{\alpha_{i}}{\alpha_{j}}\right)=\prod_{j=1, j \neq i}^{n}\left(1-r^{i-j}\right) .
$$

We may also note that the equalities obtained for $P_{i}$ represent here a special case and worth mentioning such as

$$
\sum_{i=1}^{n} \frac{1}{\prod_{j=1, j \neq i}^{n}\left(1-r^{i-j}\right)}=1 .
$$

\section{Conclusion}

The pdf and cdf and some related properties of the hypoexponential distribution with distinct parameters were established. The proofs have been done by using Laplace transform and moment generating function technique. Also with the help of some known computational theorems as Heaviside expansion theorem and multinomial expansion formula the $k^{\text {th }}$ order derivative of $f_{S_{n}}$ and the moment of this distribution of order $k$ were established, in addition for some new related equalities. Eventually, the pdf for models when the parameters $\alpha_{i}$ are arithmetic and geometric were presented. However the other two cases for hypoexponential distribution when the parameters are equal or not all equal can be studied and observed for future studies. It may be checked if they have the same properties as in this paper.

\section{REFERENCES}

[1] W. Feller, "An Introduction to Probability Theory and Its Applications," Vol. II, Wiley, New York, 1971.

[2] S. M. Ross, "Introduction to Probability Models," 10th Edition, Academic Press, San Diego, 2011.

[3] B. Anjum and H. G. Perros, "Adding Percentiles of Erlangian Distributions," IEEE Communications Letters, Vol. 15, No. 3, 2011, pp. 346-348. doi:10.1109/LCOMM.2011.011011.102143

[4] K. S. Trivedi, "Probability and Statistics with Reliability, Queuing and Computer Science Applications," 2nd Edition, John Wiley \& Sons, Hoboken, 2002.

[5] W. Kordecki, "Reliability Bounds for Multistage Structure with Independent Components," Statistics \& Probability Letters, Vol. 34, No. 1, 1997, pp. 43-51. doi:10.1016/S0167-7152(96)00164-2

[6] A. M. Mathai, "Storage Capacity of a Dam with Gamma Type Inputs," Annals of the Institute of Statistical Mathematics, Vol. 34, No. 1, 1982, pp. 591-597. doi:10.1007/BF02481056

[7] L. D. Minkova, "Insurance Risk Theory," Lecture Notes, TEMPUS Project SEE Doctoral Studies in Mathematical Sciences, 2010.

[8] G. E. Willmot and J. K. Woo, "On the Class of Erlang Mixtures with Risk Theoretic Applications," North American Actuarial Journal, Vol. 11, No. 2, 2007, pp. 99-115. doi:10.1080/10920277.2007.10597450

[9] G. Bolch, S. Greiner, H. Meer and K. Trivedi, "Queueing Networks and Markov Chains: Modeling and Performance Evaluation with Computer Science Applications," 2nd Edition, Wiley-Interscience, New York, 2006.

[10] O. Gaudoin and J. Ledoux, "Modélisation Aléatoire en Fiabilité des Logiciels," Hermès Science Publications, Paris, 2007.

[11] S. Nadarajah, "A Review of Results on Sums of Random Variables," Acta Applicandae Mathematicae, Vol. 103, No. 2, 2008, pp. 131-140. doi:10.1007/s10440-008-9224-4

[12] M. Akkouchi, "On the Convolution of Exponential Dis- 
tributions," Chungcheong Mathematical Society, Vol. 21, No. 4, 2008, pp. 501-510.

[13] S. V. Amari and R. B. Misra, "Closed-Form Expression for Distribution of the Sum of Independent Exponential Random Variables," IEEE Transactions on Reliability, Vol. 46, No. 4, 1997, pp. 519-522. doi:10.1109/24.693785

[14] Z. Jelinski and P. B. Moranda, "Software Reliability Research," Statistical Computer Performance Evaluation,
Academic Press, New York, 1972, pp. 465-484.

[15] P. B. Moranda, "Event-Altered Rate Models for General Reliability Analysis," IEEE Transactions on Reliability, Vol. R-28, No. 5, 1979, pp. 376-381. doi:10.1109/TR.1979.5220648

[16] M. R. Spiegel, "Schaum's Outline of Theory and Problems of Laplace Transforms," Schaum, New York, 1965. 